\title{
Subungual Onycholemmal Cysts: A Case Report
}

\author{
Erika Lydrup ${ }^{a} \quad$ Anette Pedersen Pilt ${ }^{b} \quad$ Volker-Jürgen Schmidt ${ }^{a}$ \\ Hannah Trøstrup ${ }^{a}$ \\ aDepartment of Plastic Surgery and Breast Surgery, Zealand University Hospital, Roskilde, \\ Denmark; bepartment of Pathology, Zealand University Hospital, Roskilde, Denmark
}

\section{Keywords}

Subungual onycholemmal cysts · Subungual epidermoid cysts · Subungual epidermoid inclusions

\section{Abstract}

Subungual onycholemmal cysts (SOCs) are rare nail abnormalities. The clinical findings vary and include onychodystrophy, ridging, nail bed pigmentation, and thickening, but most often SOCs do not cause any symptoms and are accidental findings. In this case report, we present a case of a woman with pigmentation of the toenail, suspect for melanoma. Surprisingly, the histopathological examination showed SOCs. We discuss the histological features of SOCs, etiology, and differential diagnoses.

\section{Introduction}

In this case report, a patient with pigmentation of the toenail, suspect for melanoma, is presented. Surprisingly, the histopathological examination revealed subungual onycholemmal cysts (SOCs), a rare and distinctive nail abnormality. The current casuistic addresses the histological features of SOC, etiology, and differential diagnoses.

\section{Case Report}

A 45-year-old woman was referred to our department due to pigmentation of the right great toenail, observed 3 months before. She had lost the nail as a child, due to trauma, but could not recall any recent trauma, pain, or bleeding. She had no previous history or family risk of melanoma. 
Fig. 1. Clinical photograph. The arrow indicates the lesion of the right great toenail.

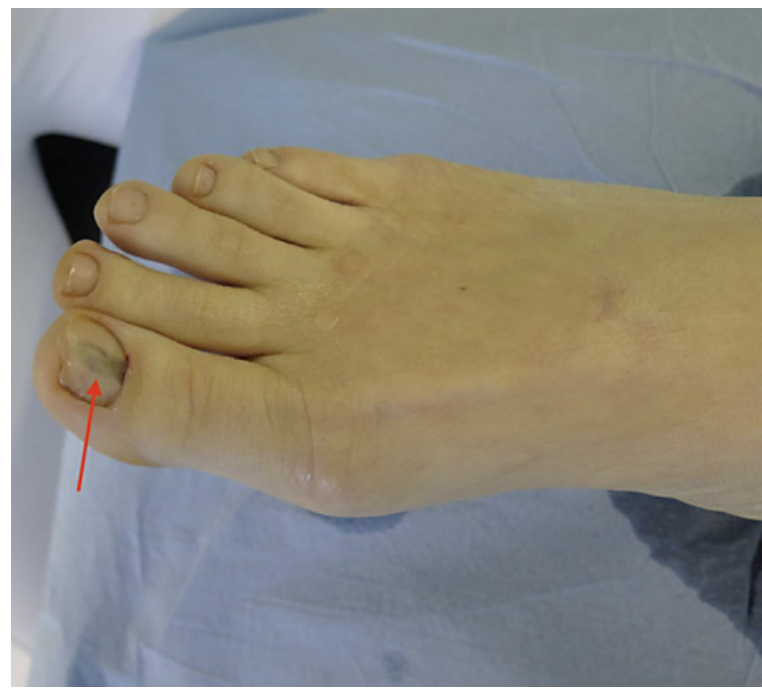

Physical examination showed a longitudinal dark streak of $15 \times 6 \mathrm{~mm}$ on the right great toenail (shown in Fig. 1). On removing the nail plate, a cystic lesion amounting $10 \times 10 \mathrm{~mm}$ appeared within the nail bed. However, pigmentation of the nail or nail bed was lacking. The remaining toenails and fingernails were unaffected. A biopsy of the nail bed was performed. Histopathological examination of the totally embedded biopsy revealed multiple cysts within the dermis of the nail bed, appearing to be free-lying cysts, but in close proximity to the epithelium of the nail bed (shown in Fig. 2, 3). The cysts were lined by the stratified squamous epithelium without the granular layer or any cellular atypia. The cysts contained luminal onycholemmal keratin (Fig. 3), whereas melanocytic lesion was not present, confirmed by negative immunohistochemical melanocytic antigens. Postoperatively, the defect healed by secondary intention (moist wound treatment) without nail deformity.

\section{Discussion}

SOCs, also known as subungual epidermoid cysts or subungual epidermoid inclusions, are rare nail abnormalities. Most often, SOCs do not cause any symptoms and are incidental findings when excision is performed for other reasons, for example, when investigating for melanoma, as in our case [1].

Subungual melanoma (SM) is the most severe differential diagnosis in SOCs. Two-thirds to three-quarters of SMs are pigmented, and thus, pigmented lesions of the nail are highly suspicious of SM. However, many of SMs are still initially misdiagnosed, and the correct diagnosis is not made until the tumor becomes thick. The diagnosis of amelanotic SM is even more challenging [2].

The clinical presentation of pigmented SM is a longitudinal brown streak of the nail originating from a sterile or germinal (nail fold) matrix. It is not the intensity of the color that is crucial for diagnosis but the structure of the pigmentation. In melanoma, the band and pigmentation are asymmetric and irregular, and the band might be wider at the proximal end than at the distal end, indicating tumor growth. Another sign of advanced melanoma is nail dystrophy, hinting that the tumor has caused thinning of the nail epithelium [1]. With adultonset streaky nail pigmentation, SM should always be excluded before any other diagnosis is 
Fig. 2. Biopsy from the nail bed with SOC. * indicates cysts. H-E. $\times 50$. SOC, subungual onycholemmal cyst.

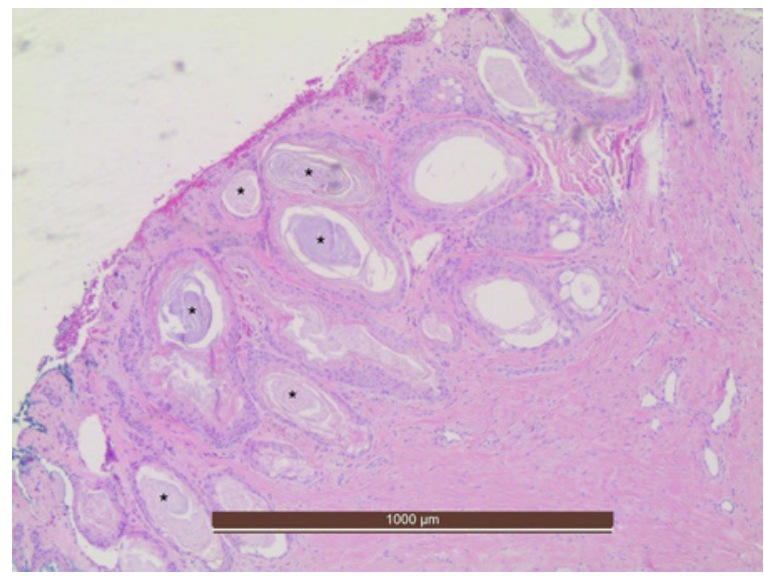

Fig. 3. Biopsy from the nail bed with SOC. * indicates cysts. Immunohistochemical positive pancytokeratin antigen, $\times 50$. SOC, subungual onycholemmal cyst.

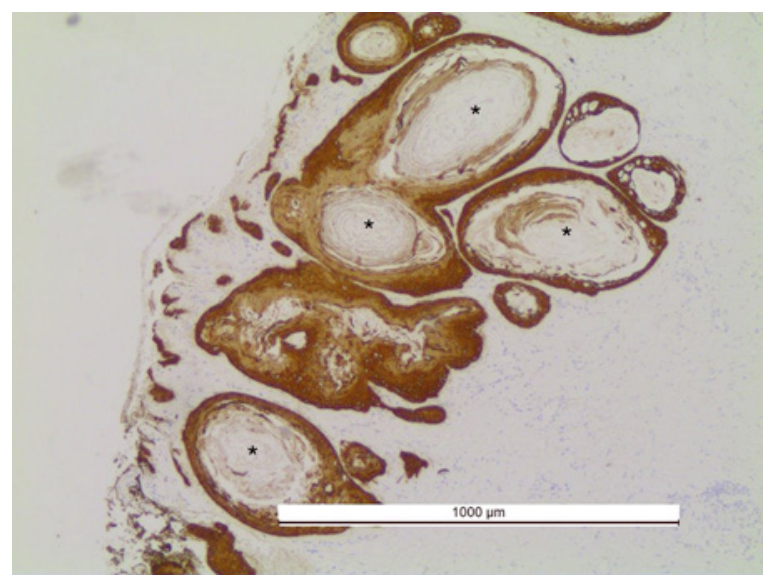

considered. A biopsy, with partial or total nail plate avulsion, is required, and the diagnosis is made by histopathological examination.

Samman [3] was the first to describe SOCs of the toenail as having a possible association with trauma. Lewin $[4,5]$ examined 90 postmortem fingernails and bed samples. Clubbing was present in 8 cases, and in all of these, SOC was detected. Lewin assumed that clubbing induces dermal fibroblast proliferation within the nail bed, leading to sequestration of the nail bed epithelium into the dermis, with subsequent cyst formation. However, microscopic SOC was also observed in normal appearing nails, lacking signs of trauma. In these cases, SOC was suggested to arise from the tips of the nail bed rete ridges, by a process of bulbous proliferation, instead of sequestration. It is tempting to speculate that SOC is a more common finding than previously assumed due its appearance in normal nails [6].

It is crucial to identify and treat signs of nail tumors as early as possible, due to the risk for malignancy, but also to prevent permanent nail deformity. The clinical findings of SOC vary and include onychodystrophy, ridging, nail bed pigmentation, thickening, or less often a normal appearing nail [6, 7]. Even though nail dystrophy is most often asymptomatic, pain is not uncommon, and often, it may be misdiagnosed for psoriasis or onychomycosis $[7,8]$.

Most commonly, SOC affects single digits, especially thumbs and great toenails, indicating that trauma could be involved in the pathogenesis [9]. It is possible that the underlying trauma occurred many years before or maybe is not recalled at all [6]. 
Appropriate diagnosis requires a nail bed biopsy, with partial or total nail avulsion. Once the diagnosis is confirmed, there is no clear treatment recommendation. However, the correct diagnosis may prevent inappropriate treatment and most importantly exclude malignancy. Moreover, if the cyst is focal, a biopsy may remove all or enough of the cyst, improving the symptoms [7]. After removal of the nail, desiccation of the nail bed should be avoided by using a nail splint or paraffin-based dressings. After large biopsies, a secondary scar removal with adequate suturing of the nail bed can prevent long-term nail deformities.

\section{Conclusion}

The etiology of SOCs is still unclear, and there is no specific treatment. However, early diagnosis of SOC may prevent an inappropriate treatment. SOC can mimic SM. In summary, SM should always be taken into consideration when a nail with pigmentation or dystrophy is present.

\section{Statement of Ethics}

The authors have no ethical conflicts to disclose. The research was conducted ethically in accordance with the World Medical Association Declaration of Helsinki. The patients gave their written informed consent, including for the use of the clinical photo.

\section{Conflict of Interest Statement}

The authors have no conflicts of interest to disclose.

\section{Funding Sources}

No funding support was obtained for this work.

\section{Author Contributions}

All authors made substantial contribution to all of the following: (1) conception and design of the work, (2) drafting the work or revising it critically for important intellectual content, (3) final approval of the version to be published, and (4) agreement to be accountable for all aspects of the work in ensuring that questions related to the accuracy or integrity of any part of the work are appropriately investigated and resolved.

\section{References}

1 Haneke E. Important malignant and new nail tumors. J Dtsch Dermatol Ges. 2017 Apr;15(4):367-86.

2 Haneke E. Ungual melanoma: controversies in diagnosis and treatment. Dermatol Ther. 2012;25(6):51024.

3 Samman PD. The human toe nail. Its genesis and blood supply. Br J Dermatol. 1959 Aug-Sep;71(8-9):296302.

4 Lewin K. The normal finger nail. Br J Dermatol. 1965;77(8):421-30.

5 Lewin K. Subungual epidermoid inclusions. Br J Dermatol. 1969 Sep;81(9):671-5.

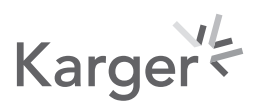


6 Busquets J, Banala M, Campanelli C, Sahu J, Lee JB. Subungual onycholemmal cyst of the toenail mimicking subungual melanoma. Cutis. 2016 Aug;98(2):107-10.

7 Telang GH, Jellinek N. Multiple calcified subungual epidermoid inclusions. J Am Acad Dermatol. 2007;56(2): 336-9.

8 Bukhari IA, Al-Mugharbel R. Subungual epidermoid inclusions. Saudi Med J. 2004 Apr;25(4):522-3.

9 Alessandor Fanti P, Tosti A. Subungual epidermoid inclusions: report of 8 cases. Dermatologica. 1989;178(4): 209-12. 